\title{
PUTTING THE STARS IN THEIR PLACES
}

\author{
SHAY ALLEN LOGAN
}

\begin{abstract}
Aвstract. This paper presents a new semantics for the weak relevant logic DW that makes the role of the infamous Routley Star more explicable. Central to this rewriting is combining aspects of both the American and Australian plan for understanding negations in relevance logics.
\end{abstract}

This IS A VERSION OF A PAPER PUBLISHED (WITH GENUINELY TERRIBLE TYPESETTING) IN Thought. Citations SHOUld, I SUPPOSE, BE DIRECTED THERE-THOUGH BE WARNED, THERE'S A SNEAKY TYPO IN THE TITLE OF THE PUBLISHED VERSION AS WELL.

\section{INTRODUCTION}

In 1974, building on work of Alasdair Urquhart, Kit Fine published a semantic theory for the usual range of sentential relevance logics. ${ }^{1}$ When Fine's semantics is mentioned in the literature, what tends to get highlighted is the technical differences between it and the better-known Routley-Meyer semantics. ${ }^{2}$ Less often noted is the fact that Fine's semantics provides an alternative philosophical perspective on relevant logics as well.

Fine interprets the points in his models as theories. This makes it clear in exactly which sense of 'logic' relevance logics are in fact logics. Specifically, relevance logics count as logic in the sense of being collections of tools we are allowed to use when building theories, no matter the subject matter of the theory or the context in which the theory building is occurring. In other words, interpreting points in models as theories suggests that the logics Fine captured can tell us what the universal theory building toolkit is. ${ }^{3}$

Of course, it's one thing to say that the points in your models are theories; it's quite another to characterize them in such a way that it becomes clear your models actually model the space of theories. In fleshing out these details, I will diverge in various ways from the technical details Fine's account. To motivate the way I will go about filling in the details, it helps to make explicit a few fairly uncontroversial assumptions I will make about theories:

(1) Theories are characterized by the sentences they take as true and the sentences they take as false. Theories, perhaps unlike states of affairs or worlds, can clearly take the same sentence to be both true and false, and can clearly refuse to classify a given sentence as either true or false.

(2) Theories can play two different roles: they can serve as foreground or as background. A typical theory (e.g. arithmetic) is constructed by applying some background theory (e.g. classical logic) to a foreground theory (e.g. the Peano Axioms). This

\footnotetext{
${ }^{1}$ See [5] and [10].

${ }^{2}$ See $[7,8,9]$

${ }^{3}$ There's room, of course, for debate about whether the universal theory building toolkit is logic, properly understood. Frankly, I don't find that debate very interesting, so I won't say anything about it other than to say that I agree with the perspective enunciated [1] and [2]: part of logic's role, traditionally, has been that of being the universal theory building toolkit. Regardless of where you stand on the matter, I take it to be a clear matter of philosophical importance to know what the universal theory building toolkit is.
} 
construction clearly iterates so that a given theory may be constructed by applying a backbackground theory $b b$ to a forebackground $f b$ theory to construct a background theory $b=b b \circ f b$ that is then applied to a foreground theory $f$ to construct the final theory $t=(b b \circ f b) \circ f$.

As in this note, we will typically use ' $\circ$ ' to stand for the operation of theory application. It seems clear that there is no reason to expect $b \circ f$ to be the same theory as $f \circ b$ nor for $(b b \circ f b) \circ f$ to be the same theory as $b b \circ(f b \circ f)$. Thus, theory application in our models will be both noncommutative and nonassociative.

(3) The way a theory behaves when being used as a background theory is determined by features of the theory itself. Since our first point was that theories are characterized by what they take to be true/false, this leads us to conclude that the way a theory behaves when being used as a background theory is governed by what it takes to be true/false

(4) Continuing the previous point, we might ask why it would be that using $b$ as a background theory and $f$ as a foreground theory gives us a theory in which $\psi$ is true. The natural sort of answer is that there is some sentence $\phi$ that $f$ takes to be true and $b$ takes it to be true that $\phi$ entails $\psi$. If we accept this explanation, it tells us something interesting: there is a type of sentence - the entailment - that governs the background-theory behavior of a given theory.

It's not clear to me how to argue for this sort of story being the right story about how background theory behavior emerges, but it's also not clear to me what alternatives there are to such a story. In any event, we will write ' $\rightarrow$ ' for entailment and suppose that entailments govern background theory related behavior.

With all of this on hand, it's fairly natural to build a semantics and see where it leads. Doing so is the purpose of the next section. The result is a semantics that captures the relevant logic DW, as we prove in the final section.

The obvious way to turn our assumptions into a semantics is by taking a model to be some sort of structure containing all of the following:

- A set of theories,

- Some apparatus or other hooking the theories up, and

- Some coherent way of spelling out what each theory takes to be true and takes to be false.

The usual way of ensuring the coherence mentioned in the last part is by explicitly declaring only the atomic commitments of each theory, then determining the remaining commitments recursively. This is very nearly the approach we will take. But we'll make one small change: rather than explicitly declaring the sentences a theory takes to be true and the sentences a theory takes to be false, we'll instead declare which sentences the theory takes to be true and which sentences it takes to be non-false. This change, while seemingly minor, will allow us to deal with the problematic issue of the Routley star in a fairly nice way, as we will see below.

So what we know so far about our our semantics is that a model should be some structure containing a set of theories together with some apparatus hooking the theories up, and functions that tell us which atomic sentences each theory takes to be true and which atomic sentences each theory takes to be non-false. 
The next bit of formalism is motivated by the following question: supposing $t$ is a theory, when is there a theory that takes as true exactly those things that $t$ takes as non-false and takes as non-false exactly those things that $t$ takes to be true?

The answer, it turns out, is subtle. Suppose $t$ is a theory and suppose $t^{\star}$ is a theory and also that $t^{\star}$ takes as true exactly what $t$ takes as non-false and takes as non-false exactly what $t$ takes as true. Provided it makes sense, it's clear this star operation must be an involution. What remains is to determine when it makes sense.

Suppose $t$ takes $\phi \vee \psi$ to be true. Then, by DeMorgan features of $\vee, t$ must also take $\neg(\neg \phi \wedge \neg \psi)$ to be true. Thus $t$ takes $\neg \phi \wedge \neg \psi$ to be false. So $t$ does not take $\neg \phi \wedge \neg \psi$ to be non-false. So $\neg \phi \wedge \neg \psi$ is not something that $t^{\star}$ takes to be true. Of course, if $t^{\star}$ took both $\neg \phi$ and $\neg \psi$ to be true, then $t^{\star}$ (being, by assumption, a theory) would take $\neg \phi \wedge \neg \psi$ to be true, contrary to what we just said. So $t^{\star}$ must either not take $\neg \phi$ to be true or not take $\neg \psi$ to be true. But this just means that $t^{\star}$ either takes $\phi$ to be non-false or $\psi$ to be non-false. But since $t^{\star}$ takes as non-false exactly those things that $t$ takes as true, this can only be the case if either $t$ already thought $\phi$ was true or already thought $\psi$ was true.

The conclusion is that $t^{\star}$ is only a theory when $t$ is prime - that is, when it takes as true at least one disjunct of each of the disjunctions it takes as true. Thus, we must require our semantics to say that a model is some structure containing a set of theories, a specified subset of which are the prime theories, together with some apparatus hooking the theories up that includes the $\star$ operation, and that finally contains functions that tell us which atomic sentences each theory takes as true/false.

Finally, recall that our goal is to determine the universal theory building toolkit. So what we really want is to know what inferences we can always make. The way to determine this is by adding to the semantics a point $\ell$ (for $\ell$ ogic) that every theory is closed under. The sentences $\ell$ makes true thus codify the inferences usable in every theory in the model.

More formally, we say that a premodel consists of

- A set $T$,

- A designated subset $P$ of $T$,

- A point $\ell \in T$,

- A binary relation $\sqsubseteq$ on $T$,

- A binary operation $\circ$ on $T$,

- A unary operation $\star$ on $P$,

- Functions $v_{t}$ and $v_{n f}$ from $T$ to sets of atoms.

The idea, of course, is for $T$ to be the set of theories recognized by the model, $P$ to be its subset of prime theories, $\ell$ to be the theory that every other theory in the model is closed under, $\sqsubseteq$ to be the relation that holds when the truths one theory recognizes are contained in the truths another theory recognizes, o to be the operation of applying one theory (as a background theory) to another, $\star$ to be the flip-flop operation discussed above, and for $v_{t}$ and $v_{n f}$ to record the atomic sentences each theory takes as true and takes as non-false, respectively. Given that this is what the pieces are supposed to be, they should be required to behave appropriately. We call a premodel in which this happens a model. Explicitly, a model is a premodel in which all of the following occur:

M1 $\sqsubseteq$ is a partial ordering,

M2 If $s \sqsubseteq t$, then

- For all $u, u \circ s \sqsubseteq u \circ t$;

- For all $u, s \circ u \sqsubseteq t \circ u$;

- $v_{t}(s) \subseteq v_{t}(t)$; and

- $v_{n f}(t) \subseteq v_{n f}(s)$ 
M3 If $s \circ t \sqsubseteq p \in P$, then there are $s^{\prime} \in P$ and $t^{\prime} \in P$ so that

$-s \sqsubseteq s^{\prime}$ and $s^{\prime} \circ t \sqsubseteq p$ and

- $t \sqsubseteq t^{\prime}$ and $s \circ t^{\prime} \sqsubseteq p$.

M4 $v_{t}(t)=\bigcap_{t \sqsubseteq p \in P} v_{t}(p)$.

M5 If $t \circ p \sqsubseteq q$ and both $p$ and $q$ are in $P$, then $t \circ q^{\star} \sqsubseteq p^{\star}$.

M6 $v_{t}\left(p^{\star}\right)=v_{n f}(p)$ and $v_{n f}\left(p^{\star}\right)=v_{t}(p)$.

M7 $p^{\star \star}=p$

M8 $\ell \circ t=t$

Most of these conditions should be unsurprising. Clauses M3, M4, and M5 are worth saying more about.

Clause M3 characterizes the behavior of prime theories: if we apply $s$ to $t$, then decide every disjunction that arises, we end up at a theory we could have gotten to by deciding every disjunction in $s$, then applying it to $t$ or by deciding every disjunction in $t$, then applying $s$ to the result. Clause M4 forces us to accept that if every way of extending $t$ to a prime includes some atom, then $t$ itself must already include that atom, which is plausible enough given our disjunction-connected understanding of primality.

For M5, suppose $q$ extends the theory we get by applying $t$ to $p$ and suppose that when we apply $t$ to $q^{*}$ we get a theory that takes $\phi$ to be true. For this to happen, there must be some $\psi$ that $q^{*}$ takes to be true which $t$ takes to entail $\phi$. Since $q^{*}$ takes $\psi$ to be true, $q$ doesn't take $\psi$ to be false. But notice that if $p$ took $\phi$ to be false, then since $t$ takes $\psi$ to entail $\phi$ and $q$ extends the theory we get by applying $t$ to $p, q$ would in fact have to take $\psi$ to be false, which is a contradiction. So $p$ must not take $\phi$ to be false. But then $p^{*}$ takes $\phi$ to be true, and thus $p^{*}$ extends the theory we get by applying $t$ to $q^{*}$.

Finally, we give our complete account of what, besides atoms, theories take to be true $\left(F_{t}\right)$ and take to be non-false $\left(F_{n f}\right)$ using the following clauses:

- $t \vDash_{t} A$ iff $A \in v_{t}(t)$ for $A$ atomic.

- $t F_{n f} A$ iff $A \in v_{n f}(t)$ for $A$ atomic.

- $t \mathrm{k}_{t} \phi \wedge \psi$ iff $t \mathrm{~F}_{t} \phi$ and $t \mathrm{k}_{t} \psi$.

- $t \vDash_{n f} \phi \wedge \psi$ iff for some $t \sqsubseteq p \in P, p \vDash_{n f} \phi$ and $p \vDash_{n f} \psi$.

- $t \vDash_{t} \phi \vee \psi$ iff for all $t \sqsubseteq p \in P, p \vDash_{t} \phi$ or $p \vDash_{t} \psi$.

- $t k_{n f} \phi \vee \psi$ iff $t k_{n f} \phi$ or $t k_{n f} \psi$.

- $t \vDash_{t} \neg \phi$ iff for all $t \sqsubseteq p \in P, p \nvdash_{n f} \phi$

- $t \vDash_{n f} \neg \phi$ iff for some $t \sqsubseteq p \in P, p \nvdash_{t} \phi$

- $t \mathrm{k}_{t} \phi \rightarrow \psi$ iff whenever $u \mathrm{~F}_{t} \phi, t \circ u \mathrm{~F}_{t} \psi$, and

- $t \vDash_{n f} \phi \rightarrow \psi$ iff for some $t \sqsubseteq p \in P$, for all $q \in P$ and $r \in P$, if $p^{\star} \circ r^{\star} \sqsubseteq q^{\star}$ and $r \vDash_{n f} \phi$, then $q \vDash_{n f} \psi$.

The first six clauses are fairly unobjectionable. They also display a pleasing symmetry that is further evidence in favor of the approach being demonstrated. For the negation clauses, one can read them as saying the following:

- $t$ is already committed to $\phi$ being false when one cannot settle the matters $t$ raises while also accepting $\phi$ as true.

- $t$ is fails to falsify $\neg \phi$ just if there is some way of settling the matters that $t$ raises that that leaves open the possibility that $\phi$ is true.

The ' $t$ ' clause for entailments is straightforward and simply details the work we expect entailments to do to ensure theories can play their background role when needed. 
Finally, we turn to the lone clause featuring the star operation: the ' ${ }_{n f}$ ' clause for entailments. Despite its scary appearance, the clause is essentially the dual to the ' $t$ ' clause for entailments. We want to detect $\phi \rightarrow \psi$ among the non-falsehoods of $t$ by looking for it among the truths of $t^{\star}$. But $t^{\star}$ may not make sense, so we look instead at the star of a prime extension of $t$. And we detect the presence of $\phi \rightarrow \psi$ by applying our theory to theories that make $\phi$ true and seeing whether they make $\psi$ true. But, modulo stars, that's exactly what the clause says. ${ }^{4}$

We round out the semantics by saying that a model validates a sentence when the distinguished point $\ell$ in the model makes the sentence true and that a sentence is valid when it is validated by every model.

So much for semantics. As it turns out, we can give a fairly nice Hilbert-system description of the valid formulas. Explicitly, all and only the formulas provable from the following axioms and rules are valid:
Axioms:
(9) $\neg \neg \phi \rightarrow \phi$
(1) $\phi \rightarrow \phi$
(2) $\phi \rightarrow(\phi \vee \psi)$
(3) $\psi \rightarrow(\phi \vee \psi)$
(4) $(\phi \wedge \psi) \rightarrow \phi$
(5) $(\phi \wedge \psi) \rightarrow \psi$
(6) $[\phi \wedge(\psi \vee \lambda)] \rightarrow[(\phi \wedge \psi) \vee(\phi \wedge \lambda)]$
(7) $[(\phi \rightarrow \psi) \wedge(\phi \rightarrow \lambda)] \rightarrow[\phi \rightarrow(\psi \wedge \lambda)]$
(8) $[(\phi \rightarrow \psi) \wedge(\lambda \rightarrow \psi)] \rightarrow[(\phi \vee \lambda) \rightarrow \psi]$
(10) $(\phi \rightarrow \neg \psi) \rightarrow(\psi \rightarrow \neg \phi)$
Rules:
(1) $\frac{\phi \quad \phi \rightarrow \psi}{\psi}$
(2) $\frac{\phi \quad \psi}{\phi \wedge \psi}$
(3) $\frac{\phi \rightarrow \psi \quad \lambda \rightarrow \gamma}{(\psi \rightarrow \lambda) \rightarrow(\phi \rightarrow \gamma)}$

We prove this assertion in the final section of the paper. Before doing so, let's reflect on what we've learned. There are a few points to be made.

First, we've given a plausible defense of the logic just described as the universal theory building toolkit. It happens to be the case that the logic described by the above axioms and rules is well-known to relevance logicians as DW. So we've learned the DW is the universal theory building toolkit.

We've also learned that DW is important for relevance-free reasons. This is good news the concept of relevance has taken a beating in recent years, so alternate reasons for being interested in these logics are (or at least should be) very welcome.

Finally, we've given a semantics that, while still using the Routley star, uses it in a pretty innocuous way. In particular, we don't need to appeal to notions of incompatibility or 'perp' (a la [3] or [4]) to explain the role the stars are playing. Again, this should be welcome in the community.

\section{Metatheory}

Lemma 1. If $t \mathrm{~F}_{t} \phi$ and $t \sqsubseteq u$, then $u \mathrm{~F}_{t} \phi$ and if $t \mathrm{~F}_{n f} \phi$ and $u \sqsubseteq t$, then $u \mathrm{~F}_{n f} \phi$.

Lemma 2. If $p \vDash_{t} \phi$ for all $t \sqsubseteq p \in P$, then $t \vDash_{t} \phi$ and if $p \vDash_{n f} \phi$ for some $t \sqsubseteq p \in P$, then $t \vDash_{n f} \phi$.

Both lemmas are proved by straightforward induction on $\phi$. We will tend to use them without comment.

Lemma 3. For $p \in P, p \vDash_{t} \phi$ iff $p^{\star} \vDash_{n f} \phi$ and $p \vDash_{n f} \phi$ iff $p^{\star} \vDash_{t} \phi$.

\footnotetext{
${ }^{4}$ The form of this clause was heavily influenced by [6], though Routley sought to completely avoid stars, where I seek only to make their role a bit more transparent.
} 
Proof. By induction on $\phi$. Most cases are straightforward; the entailment cases, which we examine, are the exception:

Let $p \vDash_{t} \phi \rightarrow \psi$. Let $q \in P, r \in P, p^{\star \star} \circ r^{\star} \sqsubseteq q^{\star}$, and $r \vDash_{n f} \phi$. Since $p^{\star \star}=p$, $p^{\star \star} \vDash_{t} \phi \rightarrow \psi$. By the inductive hypothesis (IH), $r^{\star} F_{t} \phi$. So $q^{\star} F_{t} \psi$. Thus by IH $q \vDash_{n f} \psi$. So $p^{\star} F_{n f} \phi \rightarrow \psi$. Essentially the same argument shows that if $p^{\star} F_{t} \phi \rightarrow \psi$, then $p \vDash_{n f} \phi \rightarrow \psi$.

Let $p^{\star} F_{n f} \phi \rightarrow \psi$. Then for some $p^{\star} \sqsubseteq j$, for all $q \in P$ and $r \in P$, if $j^{\star} \circ r^{\star} \sqsubseteq q^{\star}$ and $r \vDash_{n f} \phi$, then $q \vDash_{n f} \psi$. Let $u \vDash_{t} \phi$ and $p \circ u \sqsubseteq q \in P$, Then for some $u \sqsubseteq r \in P, p \circ r \sqsubseteq q$. Thus $p \circ r^{\star \star} \sqsubseteq q^{\star \star}$. Since $u \sqsubseteq r, r \vDash_{t} \phi$. So by IH, $r^{\star} \vDash_{n f} \phi$. Since $p^{\star} \sqsubseteq j, j^{\star} \sqsubseteq p$. So $j^{\star} \circ r^{\star \star} \sqsubseteq p \circ r^{\star \star} \sqsubseteq q^{\star \star}$, and thus $q^{\star} F_{n f} \phi$. So by IH, $q \vDash_{t} \phi$. Since $q$ was an arbitrary prime extension of $p \circ u, p \circ u \vDash_{t} \phi$, establishing that $p \vDash_{t} \phi \rightarrow \psi$. Essentially the same argument shows that if $p \vDash_{n f} \phi \rightarrow \psi$, then $p^{\star} \vDash_{t} \phi \rightarrow \psi$.

Theorem 1. If $\phi$ is provable then $\phi$ is valid.

Proof. By induction on the proof of $\phi$. Everything is essentially trivial, so left to the reader.

We now turn to completeness. First we need some definitions:

- If $\phi$ is provable, then we write $\vdash \phi$. We extend this by saying that $X \vdash \phi$ iff for some sequence $\psi_{1}, \psi_{2}, \ldots, \psi_{n}=\phi$, for $1 \leq i \leq n$, either (i) $\psi_{i} \in X$, or (ii) for some $j<i$ and $k<i, \psi_{i}=\psi_{j} \wedge \psi_{k}$, or (iii) for some $j<i, \vdash \psi_{j} \rightarrow \psi_{i}$.

- Say the set of formulas $X$ is a formal theory when $X \vdash \phi$ only if $\phi \in X$.

- Say the set of formulas $X$ is prime when $\phi \vee \psi \in X$ only if $\phi \in X$ or $\psi \in X$.

- Say the set of formulas $X$ is closed under disjunction when $\phi \in X$ and $\psi \in X$ only if $\phi \vee \psi \in X$.

- Write $\langle X\rangle$ for $\{\phi: X \vdash \phi\}$.

- Write $X \cdot Y$ for $\{\psi: \phi \rightarrow \psi \in X$ and $\phi \in Y\}$

- Write $X^{*}$ for $\{\phi: \neg \phi \notin X\}$.

- Write $a_{t}$ for the function $X \mapsto\{p: p$ is an atom and $p \in X\}$.

- Write $a_{n f}$ for the function $X \mapsto\{p: p$ is an atom and $\neg p \notin X\}$.

We also need the following facts, whose proofs are straightforward and omitted:

Fact $1 \vdash \neg(\phi \wedge \psi) \leftrightarrow \neg \phi \vee \neg \psi$.

Fact $2 \vdash \neg(\phi \vee \psi) \leftrightarrow \neg \phi \wedge \neg \psi$.

Fact $3 \vdash \phi \rightarrow \neg \neg \phi$.

Fact 4 If $\{\phi\} \vdash \psi$, then $\vdash \phi \rightarrow \psi$.

Fact 5 If $\vdash\left(\gamma_{1} \wedge \phi\right) \rightarrow \delta_{1}$ and $\vdash\left(\gamma_{2} \wedge \psi\right) \rightarrow \delta_{2}$, then $\vdash\left(\gamma_{1} \wedge \gamma_{2} \wedge(\phi \vee \psi)\right) \rightarrow\left(\delta_{1} \vee \delta_{2}\right)$.

Fact 6 If $\vdash \phi_{1} \rightarrow\left(\phi_{2} \rightarrow \phi_{3}\right)$ and $\vdash \psi_{1} \rightarrow\left(\psi_{2} \rightarrow \psi_{3}\right)$, then $\vdash\left(\phi_{1} \vee \psi_{1}\right) \rightarrow\left[\left(\phi_{2} \wedge \psi_{2}\right) \rightarrow\left(\phi_{3} \vee \psi_{3}\right)\right]$.

The next three lemmas are each proved by straightforward inductions on the length of the witnessing proof. The proofs are omitted.

Lemma 4. For any set of formulas $X,\langle X\rangle$ is a theory.

Lemma 5. If $t_{1}$ and $t_{2}$ are theories, then $t_{1} \cdot t_{2}$ is also a theory

Lemma 6. If $p$ is a prime theory, then so is $p^{*}$.

Lemma 7 (Lindenbaum). If $\Delta$ is closed under disjunction, $t$ is a theory and $t \cap \Delta=\emptyset$, then there is a prime theory $t^{\prime} \supseteq t$ with $t^{\prime} \cap \Delta=\emptyset$.

Proof. Let $\phi_{0} \vee \psi_{0}, \phi_{1} \vee \psi_{1}, \ldots$ be an enumeration of the disjunctions in the language. Define sets $t_{j}^{i}$ as follows: 
- $t_{0}^{0}=t$

- If $t_{j}^{i} \nvdash \phi_{j} \vee \psi_{j}$, then $t_{j+1}^{i}=t_{j}^{i}$; otherwise

- $t_{j+1}^{i}= \begin{cases}t_{j}^{i} \cup\left\{\phi_{j}\right\} & \text { if }\left\langle t_{j}^{i} \cup\left\{\phi_{j}\right\}\right\rangle \cap \Delta=\emptyset \\ t_{j}^{i} \cup\left\{\psi_{j}\right\} & \text { otherwise }\end{cases}$

- $t_{0}^{i+1}=\bigcup_{j=0}^{\infty} t_{j}^{i}$

Let $t^{\prime}=\cup_{i=0}^{\infty} t_{0}^{i}$. Seeing that $t^{\prime}$ is prime and a theory is straightforward. Suppose $t^{\prime} \cap \Delta \neq \emptyset$. Then there are $i$ and $j$ so that $\left\langle t_{j}^{i}\right\rangle \cap \Delta \neq \emptyset$. Let $i_{0}$ be the least $i$ so that for some $j,\left\langle t_{j}^{i}\right\rangle \cap \Delta \neq \emptyset$ and let $j_{0}$ be the least $j$ so that $\left\langle t_{j}^{i_{0}}\right\rangle \cap \Delta \neq \emptyset$. Note that by construction, $j_{0} \neq 0$.

If $t_{j_{0}-1}^{i_{0}} \nvdash \phi_{j_{0}-1} \vee \psi_{j_{0}-1}$, then $t_{j_{0}-1}^{i_{0}}=t_{j_{0}}^{i_{0}}$. But then since $\left\langle t_{j_{0}}^{i_{0}}\right\rangle \cap \Delta \neq \emptyset,\left\langle t_{j_{0}-1}^{i_{0}}\right\rangle \cap \Delta \neq \emptyset$, contradicting the minimality of $j_{0}$. So $t_{j_{0}-1}^{i_{0}} \vdash \phi_{j_{0}-1} \vee \psi_{j_{0}-1}$.

If $\left\langle t_{j_{0}-1}^{i_{0}} \cup\left\{\phi_{j_{0}-1}\right\}\right\rangle \cap \Delta=\emptyset$, then $t_{j_{0}}^{i_{0}}=t_{j_{0}-1}^{i_{0}} \cup\left\{\phi_{j_{0}-1}\right\}$. But $\left\langle t_{j_{0}}^{i_{0}}\right\rangle \cap \Delta \neq \emptyset$, so then $\left\langle t_{j_{0}}^{i_{0}}\right\rangle \cap \Delta=\left\langle t_{j_{0}-1}^{i_{0}} \cup\left\{\phi_{j_{0}-1}\right\}\right\rangle \cap \Delta \neq \emptyset$, a contradiction. Thus $\left\langle t_{j_{0}-1}^{i_{0}} \cup\left\{\phi_{j_{0}-1}\right\}\right\rangle \cap \Delta \neq \emptyset$. It follows that $t_{j_{0}}^{i_{0}}=t_{j_{0}-1}^{i_{0}} \cup\left\{\psi_{j_{0}-1}\right\}$. And again, since $\left\langle t_{j_{0}}^{i_{0}}\right\rangle \cap \Delta \neq \emptyset$, we have that $\left\langle t_{j_{0}}^{i_{0}}\right\rangle \cap \Delta=\left\langle t_{j_{0}-1}^{i_{0}} \cup\left\{\psi_{j_{0}-1}\right\}\right\rangle \cap \Delta \neq \emptyset$.

Altogether, we've now gathered the following information about $t_{j_{0}-1}^{i_{0}}$ :

(1) $t_{j_{0}-1}^{i_{0}} \vdash \phi_{j_{0}-1} \vee \psi_{j_{0}-1}$,

(2) $\left\langle t_{j_{0}-1}^{i_{0}} \cup\left\{\phi_{j_{0}-1}\right\}\right\rangle \cap \Delta \neq \emptyset$, and

(3) $\left\langle t_{j_{0}-1}^{i_{0}} \cup\left\{\psi_{j_{0}-1}\right\}\right\rangle \cap \Delta \neq \emptyset$.

From (2) and the finitude of proofs we see that there is $D_{1} \in \Delta$ and $\tau_{1} \in\left\langle t_{j_{0}-1}^{i_{0}}\right\rangle$ so that $\tau_{1} \wedge \phi_{j_{0}-1} \vdash D_{1}$. Similarly, (3) gives $D_{2} \in \Delta$ and $\tau_{2} \in\left\langle t_{j_{0}-1}^{i_{0}}\right\rangle$ so that $\tau_{2} \wedge \psi_{j_{0}-1} \vdash D_{2}$. Applying Fact 4 to both of these conclusions we see that $\vdash\left(\tau_{1} \wedge \phi_{j_{0}-1}\right) \rightarrow D_{1}$ and $\vdash\left(\tau_{2} \wedge \psi_{j_{0}-1}\right) \rightarrow D_{2}$. It follows by Fact 5 that $\vdash\left(\tau_{1} \wedge \tau_{2} \wedge\left(\phi_{j_{0}-1} \vee \psi_{j_{0}-1}\right)\right) \rightarrow\left(D_{1} \vee D_{2}\right)$.

But clearly $t_{j_{0}-1}^{i_{0}} \vdash \tau_{1} \wedge \tau_{2} \wedge\left(\phi_{j_{0}-1} \vee \psi_{j_{0}-1}\right)$. Thus $t_{j_{0}-1}^{i_{0}} \vdash D_{1} \vee D_{2}$. But since $\Delta$ is closed under disjunction, it follows from this that $\left\langle t_{j_{0}-1}^{i_{0}}\right\rangle \cap \Delta \neq \emptyset$, contradicting the minimality of $j_{0}$. So $t^{\prime}$ does not intersect $\Delta$.

Lemma 8. Let $P$ be the set of prime theories. Then for all theories $t, t=\bigcap_{t \subseteq p \in P} p$.

Proof. Clearly $t \subseteq \bigcap_{t \subseteq p \in P} p$. Now suppose $\phi \notin t$. Let $\Delta$ be the disjunctive closure of $\{\phi\}$. Then $t \cap \Delta=\emptyset$. So by the Lindenbaum Lemma, there is a prime theory $t^{\prime} \supseteq t$ with $t^{\prime} \cap \Delta=\emptyset$. So $\phi \notin \bigcap_{t \subseteq p \in P} p$.

Lemma 9. $p^{* *}=p$ for all prime theories $p$.

Proof. If $\phi \in p^{* *}$, then $\neg \phi \notin p^{*}$, so $\neg \neg \phi \in p$. Thus by Axiom 9, $\phi \in p$. For the other direction, notice that if $\phi \in p$, then by Fact $3, \neg \neg \phi \in p$ as well. So $\neg \phi \notin p^{*}$, and thus $\phi \in p^{* *}$.

Lemma 10. If $T$ is the set of formal theories, and $P$ is the set of prime formal theories, then $\left\langle T, P, \subseteq, \cdot, *, a_{t}, a_{n f}, \mathrm{DW}\right\rangle$ is a model. We call this model the canonical model. 
Proof. It's clear from previous work that the canonical model is a premodel. Of conditions M1-M8, only M3 remains outstanding.

So, suppose $t \cdot u \subseteq p \in P$. Let $\Delta_{t}=\{\phi:\langle\phi\rangle \cdot u \nsubseteq p\}$ and let $\Delta_{u}=\{\phi: t \cdot\langle\phi\rangle \nsubseteq p\}$. I claim both $\Delta_{t}$ and $\Delta_{u}$ are closed under disjunction.

To see this, first suppose that $\phi$ and $\psi$ are in $\Delta_{t}$. Then $\phi \vdash \phi_{1} \rightarrow \phi_{2}$ for some $\phi_{1} \in u$ and $\phi_{2} \notin p$ and $\psi \vdash \psi_{1} \rightarrow \psi_{2}$ for some $\psi_{1} \in u$ and $\psi_{2} \notin p$. So by Fact $4, \vdash \phi \rightarrow\left(\phi_{1} \rightarrow \phi_{2}\right)$ and $\vdash \psi \rightarrow\left(\psi_{1} \rightarrow \psi_{2}\right)$. Thus by Fact $6, \vdash(\phi \vee \psi) \rightarrow\left[\left(\phi_{1} \wedge \psi_{1}\right) \rightarrow\left(\phi_{2} \vee \psi_{2}\right)\right]$. So $\left(\phi_{1} \wedge \psi_{1}\right) \rightarrow\left(\phi_{2} \vee \psi_{2}\right) \in\langle\phi \vee \psi\rangle$. And since $u$ is a theory and $\phi_{1} \in u$ and $\psi_{1} \in u, \phi_{1} \wedge \psi_{1} \in u$. So $\phi_{2} \vee \psi_{2} \in\langle\phi \vee \psi\rangle \cdot u$. But since $\phi_{2} \notin p$ and $\psi_{2} \notin p$, it follows that $\phi_{2} \vee \psi_{2} \notin p$. Thus $\langle\phi \vee \psi\rangle \cdot u \nsubseteq p$, so $\phi \vee \psi \in \Delta_{t}$.

Now suppose that $\phi$ and $\psi$ are in $\Delta_{u}$. Then there are $\phi_{1}$ and $\phi_{2}$ with $\phi \vdash \phi_{1}, \phi_{1} \rightarrow \phi_{2} \in t$ and $\phi_{2} \notin p$ and there are $\psi_{1}$ and $\psi_{2}$ with $\psi \vdash \psi_{1}, \psi_{1} \rightarrow \psi_{2} \in t$ and $\psi_{2} \notin p$. By similar reasoning as in the previous part, this gives $\vdash \phi \rightarrow \phi_{1}, \vdash \psi \rightarrow \psi_{1}$, and $\phi_{2} \vee \psi_{2} \notin p$. Since $\vdash \phi \rightarrow \phi_{1}$ and $\vdash \psi \rightarrow \psi_{1}$, applications of Rule 3 and Axiom 1 give that $\vdash \phi \rightarrow\left(\phi_{1} \vee \psi_{1}\right)$ and $\vdash \psi \rightarrow\left(\phi_{1} \vee \psi_{1}\right)$. So applying Rule 1 and Axiom 8, $\vdash(\phi \vee \psi) \rightarrow\left(\phi_{1} \vee \psi_{1}\right)$. Thus $\phi_{1} \vee \psi_{1} \in\langle\phi \vee \psi\rangle$. By a brief argument, one sees that since $\phi_{1} \rightarrow \phi_{2}$ and $\psi_{1} \rightarrow \psi_{2}$ are in $t$, so are $\phi_{1} \rightarrow\left(\phi_{2} \vee \psi_{2}\right)$ and $\psi_{1} \rightarrow\left(\phi_{2} \vee \psi_{2}\right)$. Thus, applying an instance of Axiom 8, we see that $\left(\phi_{1} \vee \psi_{1}\right) \rightarrow\left(\phi_{2} \vee \psi_{2}\right) \in t$. Thus $p \not \phi_{2} \vee \psi_{2} \in t \cdot\langle\phi \vee \psi\rangle$, so $\phi \vee \psi \in \Delta_{u}$.

Now we apply the Lindenbaum Lemma. First, notice that by construction $t \cap \Delta_{t}=\emptyset$ and $u \cap \Delta_{u}=\emptyset$. Thus, by the Lindenbaum Lemma, there are $t^{\prime} \supseteq t$ and $u^{\prime} \supseteq u$ so that $t^{\prime} \cap \Delta_{t}=\emptyset$ and $u^{\prime} \cap \Delta_{u}=\emptyset$. But it follows from these equalities that $t^{\prime} \cdot u \subseteq p$ and $t \cdot u^{\prime} \subseteq p$ as required by M3.

Lemma 11. If $t$ is a theory and $\phi \rightarrow \psi \notin t$, then there are prime theories $p$ and $q$ so that $t \circ p \subseteq q, \phi \in p$, and $\psi \notin q$

Proof. To begin, let $p^{\prime}=\langle\phi\rangle$, and let $q^{\prime}=t \circ r^{\prime}$. By Lemma $4, p^{\prime}$ is a theory and then by Lemma 5, $q^{\prime}$ is a theory. Clearly $\phi \in r^{\prime}$. Suppose for contradiction that $\psi \in q^{\prime}$. Then for some $\alpha \in r^{\prime}, \alpha \rightarrow \psi \in t$. But then $\{\phi\} \vdash \alpha$, so by Fact $4, \vdash \phi \rightarrow \alpha$. Thus since $\vdash \psi \rightarrow \psi$, Rule 3 gives that $\vdash(\alpha \rightarrow \psi) \rightarrow(\phi \rightarrow \psi)$. Thus since $\alpha \rightarrow \psi \in t, \phi \rightarrow \psi \in t$ as well, which is a contradiction. So $\psi \notin q^{\prime}$. Extending $q^{\prime}$ and then $p^{\prime}$ to prime theories $q$ and $p$ meeting the necessary conditions is now straightforward using the Lindenbaum Lemma.

Lemma 12. In the canonical model, $t \vDash_{t} \phi$ iff $\phi \in t$ and $t \mathrm{~F}_{n f} \phi$ iff $\neg \phi \notin t$.

Proof. The proof is by induction on $\phi$. Only entailments present any difficulties.

First, suppose $\phi \rightarrow \psi \in t$ and $u \vDash_{t} \phi$. Then by IH, $\phi \in u$. So $\psi \in t \cdot u$ and by $\mathrm{IH}, t \cdot u F_{t} \psi$.

Next, suppose $\phi \rightarrow \psi \notin t$. Let $u=\langle\phi\rangle$. By Lemma 4, $u$ is a theory. Clearly $\phi \in u$. Suppose $\psi \in t \cdot u$. Then there is $\mu$ with $\phi \vdash \mu$ and $\mu \rightarrow \psi \in t$. Since $\phi \vdash \mu, \vdash \phi \rightarrow \mu$. But $\vdash \psi \rightarrow \psi$ as well, so by Rule 3, $\vdash(\mu \rightarrow \psi) \rightarrow(\phi \rightarrow \psi)$. So $\phi \rightarrow \psi \in t$ as well, which is a contradiction. Thus $\psi \notin t \cdot u$ and $t \not_{t} \phi \rightarrow \psi$. From here IH finishes the job.

Penultimately, suppose $\neg(\phi \rightarrow \psi) \notin t$. Let $\Delta$ be the disjunctive closure of $\{\neg(\phi \rightarrow \psi)\}$. Since $\neg(\phi \rightarrow \psi) \notin t, t \cap \Delta=\emptyset$. So there is a $t \subseteq p \in P$ with $p \cap \Delta=\emptyset$. So $\neg(\phi \rightarrow \psi) \notin p$. Thus $\phi \rightarrow \psi \in p^{*}$. Let $P \ni r$ Fnf $_{n f}$. Then by IH, $\neg \phi \notin r$. So $\phi \in r^{*}$ and thus by IH $r^{*} \vDash_{t} \phi$. Thus, if $p^{*} \cdot r^{*} \subseteq q^{*}$, then $\psi \in q^{*}$. So $\neg \psi \notin q$. Thus by IH $q \vDash_{n f} \psi$. So $t \vDash_{n f} \phi \rightarrow \psi$.

Finally, let $\neg(\phi \rightarrow \psi) \in t$. Suppose $t \subseteq p \in P$. Then $\neg(\phi \rightarrow \psi) \in p$. Thus $\phi \rightarrow \psi \notin p^{*}$. So by Lemma 11, there are $q \in P$ and $r \in P$ with $p^{*} \cdot r \subseteq q, \phi \in r$, and $\psi \notin q$. So $p^{*} \cdot r^{* *} \subseteq q^{* *}, r^{*} \vDash_{n f} \phi$, and $q^{*} \nvdash_{n f} \psi$. Thus $t k_{n f} \phi \rightarrow \psi$.

Theorem 2. If $\phi$ is not provable, then $\phi$ is invalid. 
Proof. Immediate from the previous two lemmas.

\section{REFERENCES}

[1] Jc Beall. There is no logical negation: True, false, both, and neither. Australasian Journal of Logic, 14(1):Article no. 1, 2017.

[2] Jc Beall. The simple argument for subclassical logic. Philosophical Issues, 28(1):30-54, 2018.

[3] Francesco Berto and Greg Restall. Negation on the australian plan. Journal of Philosophical Logic, 48(6):1119-1144, 2019.

[4] J Michael Dunn. Star and perp: Two treatments of negation. Philosophical perspectives, 7:331-357, 1993.

[5] Kit Fine. Models for entailment. Journal of Philosophical Logic, 3(4):347-372, 1974.

[6] Richard Routley. The American plan completed: Alternative classical-style semantics, without stars, for relevant and paraconsistent logics. Studia Logica, 43(1-2):131-158, 1984.

[7] Richard Routley and Robert K. Meyer. The semantics of entailment. Truth, Syntax, and Modality, pages 199-243. North-Holland Publishing Company, Amsterdam, 1972.

[8] Richard Routley and Robert K. Meyer. The semantics of entailment II. Journal of philosophical logic, 1(1):53-73, 1972.

[9] Richard Routley and Robert K. Meyer. The semantics of entailment III. Journal of philosophical logic, 1(2):192-208, 1972.

[10] Alasdair Urquhart. Semantics for relevant logics. The Journal of Symbolic Logic, 37(1):159-169, 1972. 